\title{
MARTINGALE METHOD FOR RUIN PROBABILITY IN AN AUTOREGRESSIVE MODEL WITH CONSTANT INTEREST RATE
}

\author{
HAILIANG YANG \\ Department of Statistics and Actuarial Science \\ The University of Hong Kong \\ Hong Kong \\ E-mail: hlyang@hkusua.hku.hk \\ LIHONG ZHANG \\ Department of Mathematical Finance \\ Beijing University \\ Beijing, People's Republic of China
}

\begin{abstract}
In this article, we consider a discrete-time insurance risk model. An autoregressive model is used to model both the claim process and the premium process. The probability of ruin is examined in a model with a constant interest rate. Both exponential and nonexponential upper bounds are obtained for the ruin probability of an infinite time horizon.
\end{abstract}

\section{INTRODUCTION}

Ruin probability arises in many applied probability models; for example, in queuing theory, it is the tail probability of the equilibrium waiting time. Ruin probability of the insurance risk model has been extensively studied. Compared to the classical model without investment incomes, there is a relatively smaller number of articles on ruin problems under the model with interest incomes. Sundt and Teugels [15] considered a compound Poisson model with a constant interest force. By using techniques similar to the classical model, upper and lower bounds for the ruin probability 
were obtained. Paulsen and Gjessing [14] considered a diffusion-perturbed classical risk model. Under the assumption of stochastic investment incomes, a Lundbergtype inequality was obtained. Paulsen [13] provided an excellent survey on this subject. Yang [16] considered a discrete-time risk model with a constant interest force. By using martingale inequalities, both a Lundberg-type inequality and nonexponential upper bounds for ruin probabilities were obtained.

In actuarial science, the classical models are usually based on the independency assumptions. However, because of the increasing complexity of insurance and reinsurance products, actuaries have been paying an increasing amount of attention to the modeling of dependent risks. There are two types of correlation. The first type is the correlation between lines of businesses. See the recent works by Dhaene and Goovaerts [5,6], Goovaerts and Dhaene [9], Müller [11,12], Denuit, Genest, and Marceau [3], Ambagaspitiya [1], Dhaene and Denuit [4], and $\mathrm{Hu}$ and $\mathrm{Wu}$ [10]. The second type is the correlation between the current claim and previous claims. Early contribution can be found in Gerber $[7,8]$. The latter is in the spirit of this article. In this article, we extend the results of Yang [16] to the correlated risk case. We consider a discrete-time risk model with a constant interest rate and assume that both the premiums and the claims are correlated random variables. This model can also be considered as an extension of the model in Bowers, Gerber, Hickman, Jones, and Nesbitt [2]. The main difference between the model in this article and the one in Bowers et al. [2] is that we introduce the interest incomes. Both exponential and nonexponential upper bounds for the ruin probability are obtained. The usefulness of the upper bounds obtained in this article and the relationship between the parameters of the model and the ruin probabilities are illustrated by some numerical examples.

The article is organized as follows. Section 2 presents the model and some assumptions. A Lundberg-type inequality is given in Section 3, and Section 4 contains the nonexponential bounds. In Section 5, some numerical results are included to illustrate the accuracy of the bounds obtained in this article.

\section{THE MODEL}

In classical risk models, we usually assume that the premiums are the same in different but equal length periods and the total amounts of claims in different periods are independent random variables. In many cases, this assumption may be unrealistic. Bowers et al. [2] considered an autoregressive model for the insurer's claim costs. Gerber [8] assumed that the surplus process could be written as an initial surplus plus the annual gains and it used a linear model to model the annual gains. The ruin probability was considered in that article by using a martingale argument. Similar work can also be found in Gerber [7]. In this article, we extend previous models by using an autoregressive process to model both the premiums and the claims. We also include investment incomes in our model.

Suppose that $\left\{W_{1}, W_{2}, \ldots\right\}$ is a sequence of independent and identically distributed (i.i.d) nonnegative random variables. Let the common distribution function of 
$W_{i}$ be $G(x)=P(W \leq x)$ and $E(W)<+\infty$, where an arbitrary $W_{i}$ is denoted by $W$. We assume that $\left\{X_{1}, X_{2}, \ldots\right\}$ is a sequence of nonnegative random variables and

$$
\begin{aligned}
& X_{k}=W_{k}+b X_{k-1}, \quad k=1,2,3, \ldots, \\
& X_{0}=x_{0},
\end{aligned}
$$

where $0 \leq b<1$. Here, $X_{i}$ denotes the premium collected during the time interval $[i-1, i]$, or the $i$ th year. We assume that the premiums at the beginning of a subsequent year are an upgrade of last year's premium plus a random noise term. One possible interpretation of this model is the following: The parameter $b$ can be interpreted as the proportion of last year's business, which will remain in this year's portfolio. The parameter $b$ measures the degree of correlation. If $b=0$, then the premium process becomes an i.i.d. random sequence and the premium collected at any time interval is independent of old information. If $b$ is close to 1 , then the process becomes very dependent. A large part of the old customers will stay in the new time period. $W_{k}$ can be thought of as the premium income in the year $k$ from the new business in the year $k$. Suppose we are at time 0 now; then, last year's premium income is known. We denote it by $x_{0}$.

In addition, we assume that $\left\{Y_{1}, Y_{2}, \ldots\right\}$ is a sequence of nonnegative random variables and

$$
\begin{aligned}
& Y_{k}=Z_{k}+a Y_{k-1}, \quad k=1,2,3, \ldots, \\
& Y_{0}=y_{0}
\end{aligned}
$$

where $\left\{Z_{k}\right\}$ is a sequence of i.i.d nonnegative random variables, independent of $\left\{W_{1}, W_{2}, \ldots\right\}$ and $0 \leq a<1$. Here, $Y_{i}$ denotes the claims during the interval $[i-1, i]$, or the $i$ th year. Similar to the premium process case, the parameter $a$ can be interpreted as the proportion of the old business in the new portfolio. At time 0, we know the claim amount of the last year. The claim amount of last year is denoted by $y_{0}$. Note that $a$ and $b$ are not necessarily equal since this is only one interpretation of the model. The model could be applied to different situations.

Let the common distribution function of $Z_{i}$ be $F(x)=P(Z \leq x)$, where an arbitrary $Z_{i}$ is denoted by $Z$ and we assume that $E Z<+\infty$. This completes the description of the first-order autoregressive models for the premium and claim processes.

Remark 1: In the above model, we assume that $a \geq 0$ and $b \geq 0$. This is necessary if we want both the premium random variables and the claim random variables to be nonnegative. In Bowers et al. [2], a first-order autoregressive process was used to model the claim process and it was assumed that the parameter is in between -1 and 1 . When the parameter is negative, it is true that the claim process may take a negative value with a positive probability. However, as long as the expected claim is positive and the probability of the claim being negative is small, the model is still a reasonable one (in the sense that it can still be used to fit the practical data and provides a reasonable approximation to the practical problems; of course, it is an 
incorrect model theoretically). In our model, if we do not require the premium and the claim to be nonnegative with a probability of 1 , we can also assume that $-1<$ $a<1$ and $-1<b<1$. All the results in this article can be extended to this case without any difficulty.

Now, we can specify the surplus process of an insurance company by the following model. Let $U_{n}$ be the surplus at time $n$ and let $r$ be the compound interest rate. Here, we assume that $r$ is a constant $(r \geq 0)$. Let $x$ denote the initial surplus. Then, the dynamic of the surplus is given by

$$
U_{n}=x(1+r)^{n}+\sum_{i=1}^{n} X_{i}(1+r)^{n-i+1}-\sum_{i=1}^{n} Y_{i}(1+r)^{n-i}
$$

Here, we assume that the claim $Y_{i}$ is paid at the end of the time period and the premium $X_{i}$ is paid at the beginning of the time period. In this article, we will also assume that the net-profit condition is true; that is,

$$
E\left[X_{i}\right]>E\left[Y_{i}\right] .
$$

This condition is equivalent to

$$
\frac{1-a^{i}}{1-a} E[Z]+a^{i} y_{0}<\frac{1-b^{i}}{1-b} E[W]+b^{i} x_{0}
$$

for all $i=1,2, \ldots$. The following condition implies condition (2) and it is easy to check:

$$
E[W]>E[Z] \text { and } b \geq a \text {. }
$$

We define the ruin probability for this model as

$$
\psi\left(x, y_{0}, x_{0}\right)=P\left(\bigcup_{n=1}^{\infty}\left(U_{n} \leq 0\right) \mid U_{0}=x, Y_{0}=y_{0}, X_{0}=x_{0}\right) .
$$

Remark 2: The above net-profit condition (2) is only a sufficient condition for the ruin probability being less than 1 . A necessary condition for the ruin probability being less than 1 is that

$$
\frac{E(Z)}{1-a v}\left[\frac{(1+r)^{n}-1}{r}-v a \frac{1-a^{n}}{1-a}\right]-\frac{E(W)}{1-b v}\left[(1+r) \frac{(1+r)^{n}-1}{r}-v b \frac{1-b^{n}}{1-b}\right]
$$

does not tend to infinity as $n \rightarrow \infty$, where $v=1 /(1+r)$.

In this article, we discuss both exponential and nonexponential upper bounds for the ruin probability.

\section{EXPONENTIAL BOUND}

Assume that the moment generating function of $Z$ exists in an appropriate region. Suppose that $a<\left(1+b v^{2}\right) / v-1$ and $E[W]>E[Z]$; we also assume that there is an $R>0$ satisfying the equation 


$$
E\left[\exp \left(-\frac{R}{1-b v} W\right)\right] E\left[\exp \left(\frac{R v Z}{1-a v}\right)\right]=1, \quad v=(1+r)^{-1}
$$

Then, $R$ is called the adjustment coefficient.

Remark 3: If $X \equiv C$ (i.e., $b=0, W_{i}=C$, and $C$ is a constant), $v=1$, and $a=0$, then $R$ is the adjustment coefficient in the simple discrete-time model without interest income and correlation.

Remark 4: Under the assumption that the moment generating function of $Z$ exists, we can prove that there is an $R>0$ satisfying Eq. (4). Note that the positive solution of Eq. (4) may not be unique. If this is the case, the adjustment coefficient is chosen as the smallest positive solution.

TheOREM 1: For $x \geq 0$,

$$
\psi\left(x, y_{0}, x_{0}\right) \leq \frac{\exp (-R \hat{x})}{E\left[\exp \left(-R v^{T} \hat{U}_{T}\right) \mid T<\infty\right]},
$$

where

$$
\begin{aligned}
\hat{U}_{n} & =U_{n}-\frac{a v}{1-a v} Y_{n}+\frac{b}{1-b v} X_{n}, \\
T & =\inf \left\{n: U_{n} \leq 0\right\} \\
\hat{x} & =\hat{U}_{0} .
\end{aligned}
$$

Inequality (5) is an equality when $r=0$.

Proof: Considering $M_{n}(x)=e^{-R v^{n} \hat{U}_{n}}$,

$$
\mathcal{F}_{n}=\sigma\left\{W_{i}, Z_{i}, i \leq n\right\}
$$

that is, $\mathcal{F}_{n}$ is a $\sigma$-field generated by $\left\{W_{i}\right\}_{i=1}^{n}$ and $\left\{Z_{i}\right\}_{i=1}^{n}$. Then,

$$
\begin{aligned}
& E\left(M_{n}(x) \mid \mathcal{F}_{n-1}\right) \\
& \quad=E\left[\exp \left(-R v^{n}\left(U_{n}-\frac{a v}{1-a v} Y_{n}+\frac{b}{1-b v} X_{n}\right)\right) \mid \mathcal{F}_{n-1}\right] \\
& \quad=E\left\{\left[\exp \left(-R v^{n} v^{-1}\left(\hat{U}_{n-1}+\frac{1}{1-b v} W_{n}-\frac{v}{1-a v} Z_{n}\right)\right)\right] \mid \mathcal{F}_{n-1}\right\} .
\end{aligned}
$$

From $\hat{U}_{n-1}$ is $\mathcal{F}_{n-1}$-measurable, the $W_{n}$ 's are independent random variables, and $Z_{n}$ 's are independent random variables, we have

$$
\begin{aligned}
& E\left(M_{n}(x) \mid \mathcal{F}_{n-1}\right) \\
& \quad=\exp \left(-R v^{n-1} \hat{U}_{n-1}\right) E\left[\exp \left(-R v^{n-1}\left(\frac{1}{1-b v} W_{n}-\frac{v}{1-a v} Z_{n}\right)\right)\right] \\
& \quad=\exp \left(-R v^{n-1} \hat{U}_{n-1}\right) E\left[\left(\exp \left(-R\left(\frac{1}{1-b v} W_{n}-\frac{v}{1-a v} Z_{n}\right)\right)\right)^{v^{n-1}}\right] .
\end{aligned}
$$


Because $v^{n-1}=(1 /(1+r))^{n-1} \leq 1$, where $r$ is the interest rate and $r \geq 0,-x^{v^{n-1}}$ $(x>0)$ is a convex function. By Jensen's inequality,

$$
\begin{gathered}
E\left[-\left(\exp \left(-R\left(\frac{1}{1-b v} W_{n}-\frac{v}{1-a v} Z_{n}\right)\right)\right)^{v^{n-1}}\right] \\
\quad \geq-\left(E\left(\exp \left(-R\left(\frac{1}{1-b v} W_{n}-\frac{v}{1-a v} Z_{n}\right)\right)\right)\right)^{v^{n-1}} .
\end{gathered}
$$

From the definition of adjustment coefficient and the assumption that $\left\{W_{1}, W_{2}, \ldots\right\}$ is independent of $\left\{Z_{1}, Z_{2}, \ldots\right\}$, we have

$$
\left(E\left(\exp \left(-R\left(\frac{1}{1-b v} W_{n}-\frac{v}{1-a v} Z_{n}\right)\right)\right)\right)^{v^{n-1}}=1 .
$$

So

$$
E\left(\left(\exp \left(-R\left(\frac{1}{1-b v} W_{n}-\frac{v}{1-a v} Z_{n}\right)\right)\right)^{v^{n-1}}\right) \leq 1
$$

and

$$
E\left(M_{n}(x) \mid \mathcal{F}_{n-1}\right) \leq \exp \left(-R v^{n-1} \hat{U}_{n-1}\right) .
$$

Then, $M_{n}(x)$ is an $\mathcal{F}_{n}$-sup-martingale (resp. a martingale when $r=0$ ).

Let $T$ be the time of ruin and let $n_{0}$ be a positive integer. Then, $T \wedge n_{0}$ is a bounded $\mathcal{F}_{n}$-stopping time. By using the Doob's bounded stopping time theorem, we have

$$
E\left(M_{0}(x)\right) \geq E\left(M_{T \wedge n_{0}}(x)\right)
$$

that is,

$$
\begin{aligned}
E\left(e^{-R \hat{x}}\right) \geq & E\left(e^{-R v^{\left(n_{0} \wedge T\right)} \hat{U}_{n_{0} \wedge T}}\right) \\
= & E\left(e^{-R v^{T} \hat{U}_{T}} \mid T \leq n_{0}\right) P\left(T \leq n_{0}\right) \\
& +E\left(e^{-R v^{n_{0}} \hat{U}_{n_{0}}} \mid T>n_{0}\right) P\left(T>n_{0}\right) \\
\geq & E\left(e^{-R v^{T} \hat{U}_{T}} \mid T \leq n_{0}\right) P\left(T \leq n_{0}\right) .
\end{aligned}
$$

Letting $n_{0} \rightarrow+\infty$, we obtain

$$
\begin{aligned}
E\left(e^{-R \hat{x}}\right) & \geq E\left(e^{-R v^{T} \hat{U}_{T}} \mid T<+\infty\right) P(T<+\infty) \\
& =E\left(e^{-R v^{T} \hat{U}_{T}} \mid T<+\infty\right) \psi\left(x, y_{0}, x_{0}\right) .
\end{aligned}
$$

From this, (5) is proved.

In Bowers et al. [2], the insurer's claim costs are modeled by an autoregressive process and the premium is assumed to be a constant over different intervals. We 
assume that $b=0$ (i.e., $X_{i}=W_{i}, i=1,2, \ldots$, are i.i.d. random variables) and we also assume that the moment generating function of $Z$ exists. We now define $R>0$ as the solution of the equation

$$
E[\exp (-R X)] E\left[\exp \left(\frac{R v Z}{1-a v}\right)\right]=1 .
$$

Then, as a special case of Theorem 1, we have the following corollary.

Corollary 1: For $x \geq 0$,

$$
\psi\left(x, y_{0}\right) \leq \frac{\exp (-R \hat{x})}{E\left[\exp \left(-R v^{T} \hat{U}_{T}\right) \mid T<\infty\right]},
$$

where

$$
\begin{aligned}
\hat{U}_{n} & =U_{n}-\frac{a v}{1-a v} Y_{n}, \\
T & =\inf \left\{n: U_{n} \leq 0\right\}, \\
\hat{x} & =\hat{U}_{0} .
\end{aligned}
$$

\section{NONEXPONENTIAL BOUND}

A problem with the above exponential bound is that when the moment generating function of $Z$ does not exist in the appropriate region, we cannot use it. In such a case, a special class of functions are used to obtain an upper bound.

We say a distribution $B(X)$ is a new worse than used (NWU) distribution if $B(x)$ is a distribution function (d.f.) of a nonnegative random variable and $\bar{B}(x)=1-B(x)$ and $\bar{B}(x) \bar{B}(y) \leq \bar{B}(x+y)$ for $x \geq 0$ and $y \geq 0$. We say that $B(x)$ is a new better than used (NBU) if $\bar{B}(x) \bar{B}(y) \geq \bar{B}(x+y)$ for $x \geq 0$ and $y \geq 0$. An important subclass of the NWU class is the class of absolutely continuous distribution with a decreasing failure rate (DFR), where $B(x)$ is DFR if the associated failure rate $-(d / d x) \ln \bar{B}(x)$ is nonincreasing in $x$.

The following result provides a nonexponential bound for the ruin probability in our setup.

Theorem 2: Suppose $x-[a v /(1-a v)] y_{0}+b(1+b v) x_{0} \geq 0, B_{1}(x)$ is a NWU d.f., $B_{2}(x)$ is a NBU d.f., and

$$
E\left\{\frac{1}{\bar{B}_{1}\left(\delta_{1} \frac{v}{1-a v} Z\right)} \bar{B}_{2}\left(\delta_{2} \frac{W}{1-b v}\right)\right\} \leq 1
$$

for all $0<\delta_{1}<1$ and $0<\delta_{2}<1$. Also assume that

$$
\bar{B}_{1}(y-x) \geq \bar{B}_{1}(y)\left\{\bar{B}_{2}(x)\right\}^{-1}, \quad y \geq x .
$$


Then,

$$
\psi\left(x, y_{0}, x_{0}\right) \leq \bar{B}_{1}\left(x-\frac{a v}{1-a v} y_{0}+b(1+b v) x_{0}\right)
$$

PROOF:

$$
\begin{aligned}
U_{n}=x(1+r)^{n} & +\sum_{k=1}^{n} X_{k}(1+r)^{n-k+1}-\sum_{k=1}^{n} Y_{k}(1+r)^{n-k} \\
=(1+r)^{n}( & x-\frac{1-(a v)^{n}}{1-a v} a v y_{0}+\frac{1-(b v)^{n}}{1-b v} b x_{0} \\
& \left.+\sum_{i=1}^{n} v^{i-1} \frac{1-(b v)^{n-i+1}}{1-b v} W_{i}-\sum_{i=1}^{n} v^{i} \frac{1-(a v)^{n-i+1}}{1-a v} Z_{i}\right) .
\end{aligned}
$$

Let

$$
H_{n}=\prod_{i=1}^{n}\left\{\frac{\bar{B}_{2}\left(v^{i-1} \frac{1-(b v)^{n-i+1}}{1-b v} W_{i}\right)}{\bar{B}_{1}\left(v^{i} \frac{1-(a v)^{n-i+1}}{1-a v} Z_{i}\right)}\right\}
$$

From Eq. (6), we know that $H_{n}$ is a supermartingale. The ruin probability

$$
\begin{aligned}
\psi\left(x, y_{0}, x_{0}\right)= & P\left\{\bigcup_{n=1}^{\infty}\left(U_{n}<0\right)\right\} \\
= & P\left\{\bigcup _ { n = 1 } ^ { \infty } \left(\sum_{i=1}^{n} v^{i} \frac{1-(a v)^{n-i+1}}{1-a v} Z_{i}-\sum_{i=1}^{n} v^{i-1} \frac{1-(b v)^{n-i+1}}{1-b v} W_{i}\right.\right. \\
& \left.\left.>x-\frac{1-(a v)^{n}}{1-a v} a v y_{0}+\frac{1-(b v)^{n}}{1-b v} b x_{0}\right)\right\} \\
\leq & P\left\{\bigcup _ { n = 1 } ^ { \infty } \left(\sum_{i=1}^{n} v^{i} \frac{1-(a v)^{n-i+1}}{1-a v} Z_{i}-\sum_{i=1}^{n} v^{i-1} \frac{1-(b v)^{n-i+1}}{1-b v} W_{i}\right.\right. \\
= & \lim _{N \rightarrow \infty} P\left\{\bigcup_{n=1}^{N}\left(\sum_{i=1}^{n} v^{i} \frac{1-(a v)^{n-i+1}}{1-a v} Z_{i}-\sum_{i=1}^{n} v^{i-1} \frac{1-(b v)^{n-i+1}}{1-a v} y_{0}+b(1+b v) x_{0}\right)\right\} \\
& >x v
\end{aligned}
$$


Since $x-[a v /(1-a v)] y_{0}+b(1+b v) x_{0}>0$, similar to Yang [16], we have $\psi\left(x, y_{0}, x_{0}\right)$

$$
\begin{aligned}
& =\lim _{N \rightarrow \infty} P\left\{\bigcup _ { n = 1 } ^ { N } \left(\left(\sum_{i=1}^{n} v^{i} \frac{1-(a v)^{n-i+1}}{1-a v} Z_{i}-\sum_{i=1}^{n} v^{i-1} \frac{1-(b v)^{n-i+1}}{1-b v} W_{i}\right)^{+}\right.\right. \\
& \left.\left.>x-\frac{a v}{1-a v} y_{0}+b(1+b v) x_{0}\right)\right\} \\
& \leq \lim _{N \rightarrow \infty} P\left\{\bigcup _ { n = 1 } ^ { N } \left(\frac{1}{\bar{B}_{1}\left(\left(\sum_{i=1}^{n} v^{i} \frac{1-(a v)^{n-i+1}}{1-a v} Z_{i}-\sum_{i=1}^{n} v^{i-1} \frac{1-(b v)^{n-i+1}}{1-b v} W_{i}\right)^{+}\right)}\right.\right. \\
& \left.\left.\geq \frac{1}{\bar{B}_{1}\left(x-\frac{a v}{1-a v} y_{0}+b(1+b v) x_{0}\right)}\right)\right\} \\
& =\lim _{N \rightarrow \infty} P\left\{\max _{1 \leq n \leq N}\left(\frac{1}{\bar{B}_{1}\left(\left(\sum_{i=1}^{n} v^{i} \frac{1-(a v)^{n-i+1}}{1-a v} Z_{i}-\sum_{i=1}^{n} v^{i-1} \frac{1-(b v)^{n-i+1}}{1-b v} W_{i}\right)^{+}\right.}\right)\right. \\
& \left.\left.\geq \frac{1}{\bar{B}_{1}\left(x-\frac{a v}{1-a v} y_{0}+b(1+b v) x_{0}\right)}\right)\right\} \\
& \leq \lim _{N \rightarrow \infty} P\left\{\max _{1 \leq n \leq N}\left(\frac{\bar{B}_{2}\left(\sum_{i=1}^{n} v^{i-1} \frac{1-(b v)^{n-i+1}}{1-b v} W_{i}\right)}{\bar{B}_{1}\left(\sum_{i=1}^{n} v^{i} \frac{1-(a v)^{n-i+1}}{1-a v} Z_{i}\right)}\right)\right. \\
& \left.\geq \frac{1}{\bar{B}_{1}\left(x-\frac{a v}{1-a v} y_{0}+b(1+b v) x_{0}\right)}\right\} \\
& \leq \lim _{N \rightarrow \infty} P\left\{\max _{1 \leq n \leq N} \prod_{i=1}^{n}\left(\frac{\bar{B}_{2}\left(v^{i-1} \frac{1-(b v)^{n-i+1}}{1-b v} W_{i}\right)}{\bar{B}_{1}\left(v^{i} \frac{1-(a v)^{n-i+1}}{1-a v} Z_{i}\right)}\right)\right. \\
& \left.\geq \frac{1}{\bar{B}_{1}\left(x-\frac{a v}{1-a v} y_{0}+b(1+b v) x_{0}\right)}\right\} \\
& \leq \phi\left(x-\frac{a v}{1-a v} y_{0}+b(1+b v) x_{0}\right) \bar{B}_{1}\left(x-\frac{a v}{1-a v} y_{0}+b(1+b v) x_{0}\right) \text {, }
\end{aligned}
$$


where $\phi$ is a function and $\phi\left(x-[a v /(1-a v)] y_{0}+b(1+b v) x_{0}\right) \leq 1$. Therefore, this theorem holds.

From Theorem 2, we have the following results.

Corollary 2: Assume $x-[a v /(1-a v)] y_{0}+b(1+b v) x_{0} \geq 0$ and suppose that $B(x)$ is a NWU d.f. and satisfies the following:

(i) $\bar{B}(y-x) \geq \bar{B}(y) e^{\mu x}$ for $y \geq x$.

(ii)

$$
\begin{aligned}
& E\left\{\frac{1}{\bar{B}\left(\delta_{1} \cdot \frac{v}{1-a v} Z\right)}\right\} E\left[e^{-\delta_{2} \mu[W /(1-b v)]}\right] \leq 1 \text { for all } 0<\delta_{1}<1 \text { and } \\
& 0<\delta_{2}<1 .
\end{aligned}
$$

Then,

$$
\psi\left(x, y_{0}\right) \leq \bar{B}\left(x-\frac{a v}{1-a v} y_{0}+b(1+b v) x_{0}\right) .
$$

Corollary 3: Suppose that $x-[a v /(1-a v)] y_{0}+b(1+b v) x_{0} \geq 0$ and $B(x)$ is a DFR d.f. with a failure rate of $\mu_{B}(x)=(d / d x) \ln \bar{B}(x)$ and $\mu=\lim _{x \rightarrow \infty} \mu_{B}(x)>0$

$$
E\left\{\frac{1}{\bar{B}\left(\delta_{1} \frac{v}{1-a v} Z\right)}\right\} E\left[e^{-\delta_{2} \mu[W /(1-b v)]}\right] \leq 1
$$

for all $0<\delta_{1}<1$ and $0<\delta_{2}<1$. Then,

$$
\psi\left(x, y_{0}\right) \leq \bar{B}\left(x-\frac{a v}{1-a v} y_{0}+b(1+b v) x_{0}\right) .
$$

\section{EXAMPLES}

In this section, we present some examples. The examples are for illustrating the tightness of the upper bounds in this article and the relationship between the parameters of the model and the ruin probabilities. Application of this model to a realworld problem and model fitting will be an interesting future research topic. We simulate the true ruin probabilities and compare them to the results obtained from the upper bounds. We will use only the numerators of the derived upper bounds. It is not difficult to check that, in our examples, the denominators of the derived upper bounds are greater than 1 . This will disclose the accuracy of the upper bounds we obtained in this article. We can see that, in most of the cases, the upper bounds are about three to five times the true ruin probabilities (except in Example 4). However, it is not easy to obtain the true ruin probability in general. The upper bounds, like the ones in this article, are very easy to obtain, and in most of the practical problems, we only need a conservative upper bound for the ruin probability. In the following 
TABLE 1. $X=1$ and $Z$ Is Weibull

\begin{tabular}{lccc}
\hline & $x=1.5$ & $x=2$ & $x=2.5$ \\
\hline$y_{0}=0$ & $0.0723(0.3048)$ & $0.0593(0.2051)$ & $0.0436(0.1380)$ \\
$y_{0}=0.1$ & $0.0726(0.3073)$ & $0.0594(0.2068)$ & $0.0436(0.1392)$ \\
$y_{0}=0.2$ & $0.0728(0.3098)$ & $0.0594(0.2085)$ & $0.0437(0.1403)$ \\
$y_{0}=0.3$ & $0.0731(0.3123)$ & $0.0596(0.2101)$ & $0.0439(0.1414)$ \\
$y_{0}=0.4$ & $0.0736(0.3148)$ & $0.0597(0.2119)$ & $0.0441(0.1426)$ \\
$y_{0}=0.5$ & $0.0741(0.3174)$ & $0.0602(0.2136)$ & $0.0442(0.1437)$ \\
$y_{0}=0.6$ & $0.0744(0.3199)$ & $0.0603(0.2153)$ & $0.0443(0.1449)$ \\
$y_{0}=0.7$ & $0.0745(0.3225)$ & $0.0605(0.2171)$ & $0.0443(0.1461)$ \\
$y_{0}=0.8$ & $0.0745(0.3251)$ & $0.0610(0.2188)$ & $0.0445(0.1473)$ \\
$y_{0}=1$ & $0.0750(0.3304)$ & $0.0615(0.2224)$ & $0.0449(0.1497)$ \\
\hline
\end{tabular}

simulations, we used 2000 time intervals so that the true ruin probability could be a little larger than the simulated results.

Example 1: Let $P(X=c)=1$, where $c$ is a positive constant. We let $c=1$ in this example. $Z$ follows a Weibull distribution with a density function given by

$$
f_{Z}(z)=2 z e^{-z^{2}} \text { for } z>0 .
$$

Let $R$ be the solution to Eq. (4). The other parameters used in this example are $r=0.08$ and $a=0.1$. Then, we have $R=0.7921$. It is easy to check that all of the required conditions are satisfied. The simulation results and upper bounds (shown in parentheses) are given in Table 1.

From Table 1, we can see that if $y_{0}$ increases when the initial surplus $x$ is fixed, then the ruin probability also increases. Since $y_{0}$ denotes the initial claim, this is just saying that when the claim is larger, the ruin probability will be larger. It is also clear that when the initial surplus increases, the ruin probability decreases.

Example 2: In this example, we assume that both the premium process and the claim process follow autoregressive models. We assume that $W$ and $Z$ are Weibull distributed. The density function for $Z$ is the same as that in Example 1 and the density function for $W$ is given by

$$
f_{W}(w)=w e^{w^{2} / 2} \quad \text { for } x>0 .
$$

Let $r$ and $a$ be the same as in Example 1 and let $b=0.1$. By solving Eq. (4), we have the adjustment coefficient $R=1.40496$. The simulation results and upper bounds (shown in parentheses) are given in Table 2.

The results from this example indicate that for a fixed initial surplus and $y_{0}$, the ruin probability decreases when $x_{0}$ increases. This is because the initial premium is 
TABLE 2. Both $X$ and $Z$ Are Weibull

\begin{tabular}{cccccc}
\hline & $x_{0}=0$ & $x_{0}=0.2$ & $x_{0}=0.4$ & $x_{0}=0.6$ & $x_{0}=0.8$ \\
\hline$x=1.5$, & 0.0417 & 0.0415 & 0.0414 & 0.0414 & 0.0411 \\
$y_{0}=0$ & $(0.1215)$ & $(0.1178)$ & $(0.1142)$ & $(0.1108)$ & $(0.1074)$ \\
$x=2$, & 0.0211 & 0.0204 & 0.0201 & 0.0200 & 0.0189 \\
$y_{0}=0$ & $(0.0602)$ & $(0.0584)$ & $(0.0566)$ & $(0.0549)$ & $(0.0532)$ \\
$x=1.5$, & & 0.0418 & 0.0414 & 0.0413 & 0.0412 \\
$y_{0}=0.2$ & & $(0.1213)$ & $(0.1173)$ & $(0.1140)$ & $(0.1105)$ \\
$x=2$, & & 0.0207 & 0.0203 & 0.0201 & 0.0194 \\
$y_{0}=0.2$ & & $(0.0601)$ & $(0.0582)$ & $(0.0565)$ & $(0.0547)$ \\
\hline
\end{tabular}

large if $x_{0}$ is large. Since the premium process follows a time-series model, $x_{0}$ being large will result in the premiums during later time periods also being large.

Example 3: Let $P(X=c)=1$, where $c$ is a positive constant. If $Z \sim N\left(\mu, \sigma^{2}\right)$, then $R$ is the solution to

$$
e^{-c R} \int_{-\infty}^{+\infty} e^{R v x /(1-a v)} \frac{1}{\sqrt{2 \pi} \sigma} e^{-(x-u)^{2} / 2 \sigma^{2}} d x=1 .
$$

We can work out that

$$
R=\frac{2(c-[u v /(1-a v)])}{\sigma^{2}(v /(1-a v))^{2}} .
$$

Let the parameters be $\mu=10, \sigma^{2}=3^{2}, r=0.08, a=0.5$, and $c=22$. Then $R=0.3557$ and all of the conditions in this article for obtaining the upper bound are satisfied. Note that the Normal distribution may take negative values; however, in our example, it has a very small probability of taking a negative value $(<1 \%)$. Also, for the Normal distribution, the adjustment coefficient $R$ has a closed-form expression. For this reason, in the literature, the Normal distribution has been used as a claim random variable distribution. An example of this is in the standard textbook by Bowers et al. [2]. The simulation results and upper bounds are given in Table 3

The results in Table 3 clearly indicate that when the initial surplus increases, the ruin probability decreases. It again shows that as $y_{0}$ increases, the ruin probabilities also increase.

Example 4: Let $P(X=c)=1$, where $c$ is a positive constant. Assume that $Z \sim$ $\operatorname{Gamma}(n, \lambda)$. Then, $R$ is the solution to

$$
e^{-c R} \int_{0}^{+\infty} \frac{1}{\Gamma(n)} e^{-\lambda x} \lambda^{n} x^{n-1} e^{R v x /(1-a v)} d x=1 ;
$$


TABLE 3. $X=22$ and $Z$ Is Normal

\begin{tabular}{cccc}
\hline & $y_{0}=0$ & $y_{0}=1$ & $y_{0}=2$ \\
\hline$x=2$ & $0.1093(0.4910)$ & $0.2576(0.6671)$ & $0.4357(0.9065)$ \\
$x=3$ & $0.0961(0.3440)$ & $0.1011(0.4675)$ & $0.2534(0.6352)$ \\
$x=4$ & $0.0551(0.2410)$ & $0.0632(0.3275)$ & $0.0741(0.4451)$ \\
$x=5$ & $0.0402(0.1689)$ & $0.0445(0.2295)$ & $0.0518(0.3119)$ \\
$x=6$ & $0.0303(0.1183)$ & $0.0384(0.1608)$ & $0.0455(0.2185)$ \\
$x=7$ & $0.0298(0.0829)$ & $0.0302(0.1127)$ & $0.0314(0.1531)$ \\
$x=8$ & $0.0238(0.0581)$ & $0.0251(0.0789)$ & $0.0316(0.1073)$ \\
\hline
\end{tabular}

that is,

$$
e^{-c R}=\frac{(\lambda-R v /(1-a v))^{n}}{\lambda^{n}} .
$$

Here, we chose $r=0.08, n=2, \lambda=4, c=1$, and $a=0.3$. Using numerical methods, we can find that $R=1.9333$. Similarly, choosing $a=0.2$, we have $R=2.5231$. The parameters used in this example, as in other examples, are for the purpose of disclosing the relationship between the upper bound and the true ruin probability, but we would also like to see the impact of the parameter $a$ on the ruin probabilities. When we chose the parameters, we checked that these numbers satisfied all the required conditions. The simulation results and upper bounds are given in Table 4 .

This example shows that the ruin probability increases when $a$ increases. This is because the claims will be large if $a$ is large (a large $a$ implies that a large proportion of the claim in the previous time period will be likely to occur in a later time period).

Example 5 (Nonexponential Bound): This example provides some numerical results obtained using the nonexponential upper bound obtained in this article. In this example, we assume that $P(X=c)=1$ and that $c$ is a positive constant. Let $Z$ be inverse Gaussian distributed with parameters $\lambda<0, \mu>0$, and $\beta>0$. Its probability density is given by

$$
f_{Z}(z)=M z^{\lambda-1} e^{-\mu z-(z / \beta)}, \quad z \geq 0 .
$$

Here, we chose $\mu=1, \beta=1$, and $\lambda=-1$. Then, $M=(0.2798)^{-1}$,

$$
\begin{aligned}
& \bar{B}_{1}(x)=(1+k x)^{-\alpha} e^{-\mu x}, \quad k>0, \alpha>0, \\
& \bar{B}_{2}(x)=e^{-\mu(1+r-a) x} .
\end{aligned}
$$

In this example, we assume that the parameters $a$ and $r$ satisfy the relation $a \geq r$. Then, $\bar{B}_{1}(x)$ is a NWU d.f. and $\bar{B}_{2}(x)$ is a NBU d.f. Moreover,

$$
\bar{B}_{1}(y-x) \geq \bar{B}_{1}(y)\left(\bar{B}_{2}(x)\right)^{-1} \text { for all } y \geq x .
$$


TABLE 4. $X=1$ and $Z$ Is Gamma

\begin{tabular}{|c|c|c|c|c|c|c|c|c|c|}
\hline & $y_{0}=0$ & $y_{0}=0.3$ & $y_{0}=0.4$ & $y_{0}=0.5$ & $y_{0}=0.6$ & $y_{0}=0.8$ & $y_{0}=0.9$ & $y_{0}=1$ & $y_{0}=1.1$ \\
\hline $\begin{array}{l}x=0.8 \\
\quad a=0.3\end{array}$ & & $\begin{array}{l}0.0323 \\
\quad(0.2662)\end{array}$ & $\begin{array}{l}0.0328 \\
\quad(0.2867)\end{array}$ & $\begin{array}{l}0.0337 \\
\quad(0.3089)\end{array}$ & $\begin{array}{l}0.0348 \\
\quad(0.3327)\end{array}$ & & & & \\
\hline $\begin{array}{c}x=0.8 \\
a=0.2\end{array}$ & & $\begin{array}{l}0.0170 \\
\quad(0.1578)\end{array}$ & $\begin{array}{l}0.0180 \\
\quad(0.1671)\end{array}$ & $\begin{array}{l}0.0200 \\
\quad(0.1770)\end{array}$ & $\begin{array}{l}0.0210 \\
\quad(0.1874)\end{array}$ & & & & \\
\hline $\begin{array}{l}x=1, \\
a=0.3\end{array}$ & $\begin{array}{l}0.169 \\
\quad(0.1447)\end{array}$ & & $\begin{array}{l}0.0231 \\
\quad(0.1948)\end{array}$ & $\begin{array}{l}0.0243 \\
\quad(0.298)\end{array}$ & $\begin{array}{l}0.0257 \\
\quad(0.2260)\end{array}$ & & & & \\
\hline $\begin{array}{l}x=1, \\
\quad a=0.2\end{array}$ & $\begin{array}{l}0.0009 \\
\quad(0.802)\end{array}$ & & $\begin{array}{l}0.0119 \\
\quad(0.1009)\end{array}$ & $\begin{array}{l}0.0135 \\
\quad(0.1068)\end{array}$ & $\begin{array}{l}0.0138 \\
\quad(0.1131)\end{array}$ & & & & \\
\hline $\begin{array}{l}x=1.2, \\
a=0.3\end{array}$ & & & & & $\begin{array}{l}0.0154 \\
\quad(0.1535)\end{array}$ & $\begin{array}{l}0.0256 \\
\quad(0.1782)\end{array}$ & $\begin{array}{l}0.0322 \\
\quad(0.1919)\end{array}$ & $\begin{array}{l}0.0361 \\
\quad(0.2067)\end{array}$ & \\
\hline $\begin{array}{l}x=1.2, \\
a=0.2\end{array}$ & & & & & $\begin{array}{l}0.0083 \\
\quad(0.0683)\end{array}$ & $\begin{array}{l}0.0087 \\
\quad(0.0766)\end{array}$ & $\begin{array}{l}0.0089 \\
\quad(0.0811)\end{array}$ & $\begin{array}{l}0.0093 \\
\quad(0.0859)\end{array}$ & \\
\hline $\begin{array}{l}x=1.5, \\
a=0.3\end{array}$ & & & & & & $\begin{array}{l}0.0109 \\
\quad(0.0997)\end{array}$ & $\begin{array}{l}0.0130 \\
\quad(0.1074)\end{array}$ & $\begin{array}{l}0.0141 \\
\quad(0.1157)\end{array}$ & $\begin{array}{l}0.0142 \\
\quad(0.1247)\end{array}$ \\
\hline $\begin{array}{l}x=1.5, \\
a=0.2\end{array}$ & & & & & & $\begin{array}{l}0.0051 \\
\quad(0.0359)\end{array}$ & $\begin{array}{l}0.0054 \\
\quad(0.0381)\end{array}$ & $\begin{array}{l}0.0055 \\
\quad(0.0403)\end{array}$ & $\begin{array}{l}0.0059 \\
(0.0427)\end{array}$ \\
\hline
\end{tabular}


TABLE 5. $X=0.45$ and $Z$ Is Inverse Gaussian

\begin{tabular}{cccc}
\hline & $y_{0}=0$ & $y_{0}=1$ & $y_{0}=3$ \\
\hline$x=2$ & $0.0771(0.1329)$ & $0.1423(0.3170)$ & $0.4719(0.7569)$ \\
$x=3$ & $0.0313(0.0485)$ & $0.0612(0.1156)$ & $0.0843(0.2759)$ \\
$x=4$ & $0.0114(0.0177)$ & $0.0272(0.0422)$ & $0.0744(0.1006)$ \\
\hline
\end{tabular}

Choose $c$ such that

$$
E\left(\frac{1}{\bar{B}_{1}\left(\delta \frac{1}{1+r-a} Z\right)} \bar{B}_{2}(\delta c)\right) \leq 1 \quad \text { for } 0<\delta<1
$$

If $k=\alpha=0.1$, we require that $c \geq 0.4372$.

If we choose $c=0.45$, then all of the conditions in Theorem 2 are satisfied. The simulation results and upper bounds when $a=0.5$ and $r=0.08$ are given in Table 5 .

The upper bound in this example provides a better bound for the ruin probability than in other examples. This at least shows that the exponential upper bound is not necessarily better than the nonexponential bound.

\section{Acknowledgment}

The work described in this article was supported by a grant from the Research Grants Council of the Hong Kong Special Administrative Region, China (Project No. HKU 7139/01H).

\section{References}

1. Ambagaspitiya, R.S. (1999). On the distributions of two classes of correlated aggregate claims. Insurance: Mathematics and Economics 24: 301-308.

2. Bowers, N.L., Gerber, H.U., Hickman, J.C., Jones, D.A., \& Nesbitt, C.J. (1997). Actuarial mathematics, 2nd ed. Schaumburg, IL: The Society of Actuaries.

3. Denuit, M., Genest, C., \& Marceau, E. (1999). Stochastic bounds on sums of dependent risks. Insurance: Mathematics and Economics 25: 85-104.

4. Dhaene, J. \& Denuit, M. (1999). The safest dependence structure among risks. Insurance: Mathematics and Economics 25: 11-21.

5. Dhaene, J. \& Goovaerts, M.J. (1996). Dependency of risks and stop-loss order. ASTIN Bulletin 26: 201-212.

6. Dhaene, J. \& Goovaevrts, M.J. (1997). On the dependency of risks in the individual life model. Insurance: Mathematics and Economics 19: 243-253.

7. Gerber, H.U. (1981). On the probability of ruin in an autoregressive model. Bulletin of the Association of Swiss Actuaries 77: 131-141.

8. Gerber, H.U. (1982). Ruin theory in the linear model. Insurance: Mathematics and Economics 1: $177-184$.

9. Goovaerts, M.J. \& Dhaene, J. (1996). The compound Poisson approximation for a portfolio of dependent risks. Insurance: Mathematics and Economics 18: 81-85. 
10. Hu, T. \& Wu, Z. (1999). On the dependence of risks and the stop-loss premiums. Insurance: Mathematics and Economics 24: 323-332.

11. Müller, A. (1997). Stochastic orderings generated by integrals: A unified study. Advances in Applied Probability 29: 414-428.

12. Müller, A. (1997). Stop-loss order for portfolios of dependent risks. Insurance: Mathematics and Economics 21: 219-223.

13. Paulsen, J. (1998). Ruin theory with compounding assets-A survey. Insurance: Mathematics and Economics 22(1): 3-16.

14. Paulsen, J. \& Gjessing, H.K. (1997). Ruin theory with stochastic return on investments. Advances in Applied Probability 29: 965-985.

15. Sundt, B. \& Teugels, J. (1995). Ruin estimates under interest force. Insurance: Mathematics and Economics 16: 7-22.

16. Yang, H. (1999). Non-exponential bounds for ruin probability with interest effect included. Scandinavian Actuarial Journal 66-79. 